\title{
A Design Method for Composition and Reuse Oriented Weaponry Model Architecture
}

\author{
Meng Zhang ${ }^{1,}$, , Hong Wang ${ }^{1}$, Yiping $\mathrm{Yao}^{1,2}$ \\ ${ }^{1}$ College of Computer, National University of Defense Technology, Changsha, 410073, China \\ ${ }^{2}$ College of Information System and Management, National University of Defense Technology, \\ Changsha, 410073, China \\ aemail: zhangmeng_sim@163.com
}

Keywords: Warfare simulation; Model composition; Model reuse; Weaponry model architecture

\begin{abstract}
Weaponry model architecture oriented to composition and reuse can improve modeling efficiency and ensure consistency of models, for developers can build weapon models by model composition and reuse. However, there are massive models in the field of military equipment, and the combination and inheritance relationship between the models are complex, which make it hard to design weaponry model architecture. A three-layer model architecture designing method is presented in this paper in view of the above difficulties. The first layer is the entity model layer, which is established by classifying the weapon models. The second layer is the function model layer, which is extracted from the capacity requirements of warfare simulation system. The third layer is the abstract entity model layer, which is the abstraction of the entity model layer and the function model layer. At the end of this paper, we prove that weapon models in this architecture can be assembled and reused through an example of aircraft modeling.
\end{abstract}

\section{Introduction}

Simulation and modeling technology is widely used to analyze warfare problems due to its security and repeatability [1]. As a typical application of M\&S technology in the field of warfare, Warfare simulation system needs to model different types of equipments and forces [2]. However, with the development of new technologies, the types of weapons are growing, and the functions are more and more complex. In order to reduce the duplication development of the same or similar functional modules and workload of equipment modeling, model reuse and composition are highly necessary for equipment modeling [3].

Weaponry model architecture is a set of equipment models and their relationships of different types and levels [4]. Composition and reuse oriented weaponry model architecture provides an unified framework for the development of the equipment models, and can ensure the consistency and interoperability of models, as a result developers can build weapon model by model composition and reuse. However, it is very difficult to set up the composition and reuse oriented weaponry model architecture, because: 1) The warfare simulation system involves numerous kinds of models, such as weapon models, sensor models and sea, land and air based platform, etc.; 2) The relationship among those equipment models are varied, such as inheritance, combination, command and control, interaction and so on. To address those demands and problems, a composition and reuse oriented weaponry model architecture designing method is presented in this paper. It has three-layer model architecture: entity model layer, function model layer and abstract entity model layer.

The reminder of the paper is organized as follows. In section 2, the related research work is presented. In section 3, the design principles of the weaponry model architecture is discussed based on the requirement of the development personnel and the users. In section 4, a composition and reuse oriented weaponry model architecture designing method is proposed based on the design principles. In section 5, an example is given to prove that we can develop equipment models by composition and reuse based on the composition and reuse oriented weaponry model architecture. In the last section, we summarize the proposed method and some future works are raised. 


\section{Related work}

As the weaponry model architecture is an important part of the warfare simulation system, lots of warfare simulation systems have been studied it, such as JWSOL [5] and JWARS [6]. In addition, some scholars have put forward the corresponding design methods, such as Dudgeon [7].

JWSOL (The Joint Warfare Simulation Object Library) is a reusable joint operations simulation model library, designed by DMSO as the delegation of Navy Command Control and Ocean Surveillance Center. JWSOL divide simulation objects into three main categories: Agent, Event and Physical. Physical is what people normally associate with "objects" — ships, boats, planes, communications equipment, etc. JWSOL uses the object-oriented [8] method to model the equipments involved in the campaign level warfare simulation system, and describes the model hierarchy according to the inheritance relationship of different kinds of equipment models.

JWARS (Joint Warfare System) use objects oriented approach to build model for the warfare space objects. Based on the BSE (Battle Space Entity) unit, JWARS uses varieties ways, such as inheritance, polymorphism and combination, to describe the relationship between battle space entities. BSE model is composed of difference functional components which contain command control, sensors, resource management, Internet, communication management etc. With this model architecture, JWARS can flexible combine weapon and equipment models by model reuse.

Dudgeon analyzed the equipment model architecture of Janus, MODSAF [9], WARSIM 200 [10] and JWARS [6] in the standpoint of platform. He proposes a model architecture used for develop standard platform level model. Figure 1 provides an overview of the model architecture. This model architecture describes the function composition of equipment models and the Inheritance relationship between them, based on object-oriented technology. In the model architecture, the equipment model can be generated by mode composition and reuse. However, Dudgeon doesn't mention any technology and principle about model function partition. He merely summarizes the model function partition of the existing warfare simulation system.

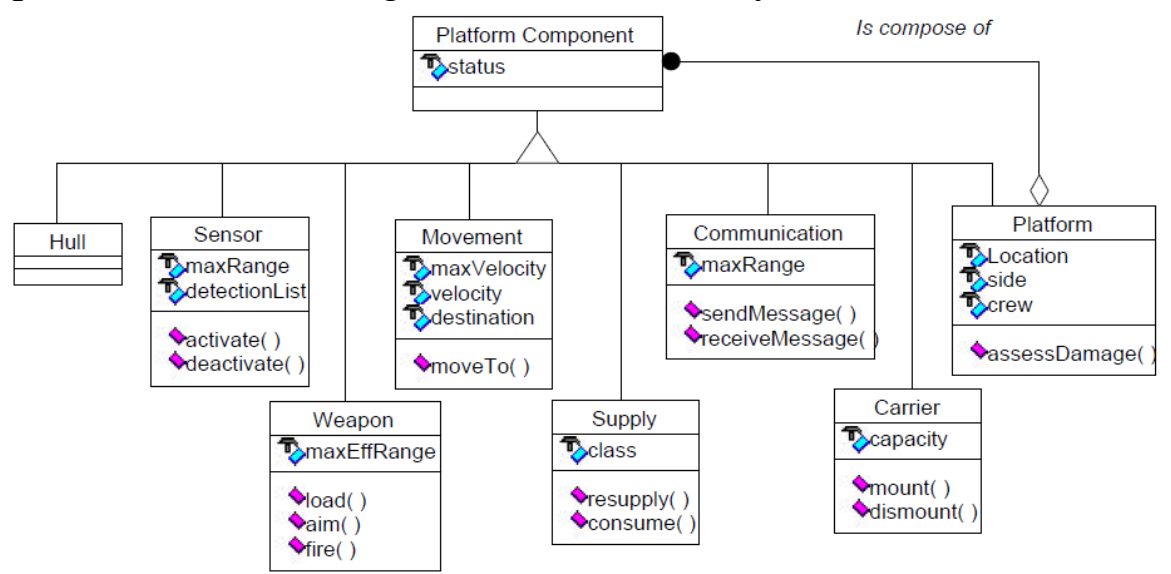

Fig. 1. Standard platform and platform Component Class

In summary, the existing warfare simulation system generally sums up public function modules based on the decomposition of the function of different equipment models. Then they use the object-oriented modeling technology construct weaponry model architecture based on the inheritance and combination relations among the function modules. However, there is no effective way to extract the public function modules of weapons and equipment, but the empirical rules. In addition, the division and abstraction of the functional modules of the weapon equipment model are also lack of Standardized technologies.

\section{Principles}

The way of the weaponry model architecture design has a great influence on the development and use of warfare simulation system. The difference of the weaponry model architecture will affect the developers' development of the weapon models, and it will also affect military experts' understanding and identification to the system. For simulation developers, weaponry model 
architecture should be able to fully reflect the model's composability and reusability. So that the weapon models can be developed in a variety of ways, thus it can avoid the duplication of modeling, and ensure the consistency of the model, and improve the development efficiency and quality of the warfare simulation system. For military personnel, the design of the weaponry model architecture should refer to the classification and combination relations of the real weaponry system and reflect the Operational capability of the real weaponry system, so as to facilitate the use of it.

In summary, the design of the weaponry model architecture should: 1) show the various equipment entities in military operations; 2) reflect the composability and reusability of the equipment model; 3) provide unified model framework.

\section{Method}

Based on design principles above, this section proposes a design method for combination and reuse oriented weaponry model architecture. From the model combination and model reuse standpoint, we design the model architecture with three-layer by this method, as shown in figure 2 . The model in the first layer is called the entity model. Based on the object-oriented technology, the first layer describes the physical model and attributes of all the equipments involved in the warfare simulation system. In order to facilitate the understanding and use for military personnel, equipment model should be consistent with the military concept model. So the establishment of the entity model layer can refer to the classification of the real weaponry. According to different operational functions, the equipment model can be divided into seven categories: sensor, weapon, communication equipment, ammunition, and sea, land, air based platform. Each category can also be further divided according to the requirements. The first layer in Figure 2 shows the entity model layer of the weaponry model architecture.

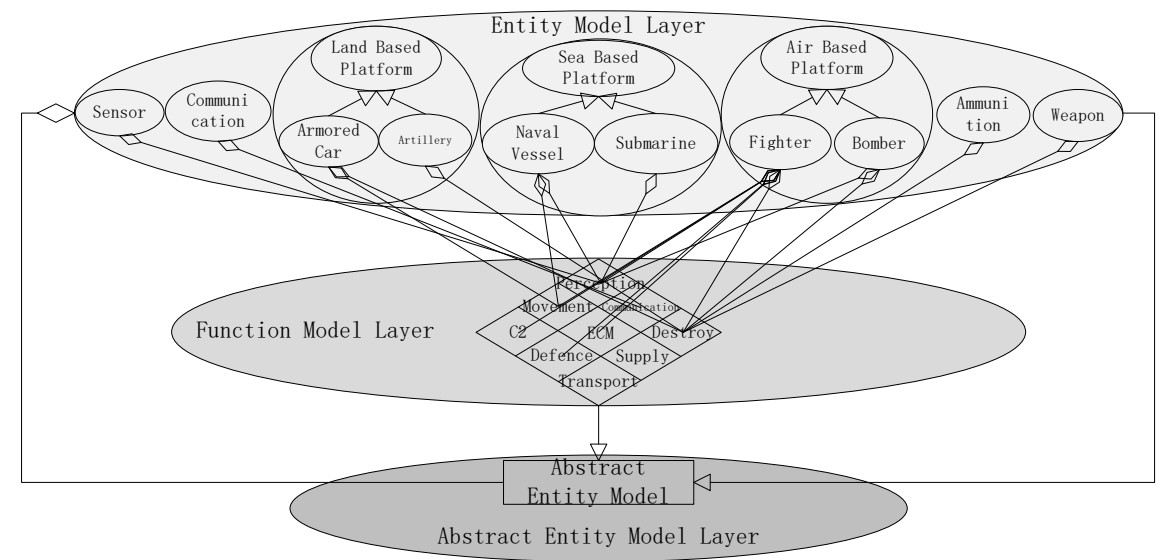

Fig. 2. The three-layer model architecture

The second layer is called function model layer. The weapon equipment is the embodiment of the operational capability, the modeling of the weapon equipment is to meet the requirement of the ability of the warfare simulation system. Generally, equipment model with different operational capability can be assembled by corresponding basic functional modules. Through analyzing the capability requirement of the warfare simulation system, we can extract the basic function module, which can meet the needs of the equipments assembly modeling. Figure 3 shows the process of extracting the basic function module from the operational capability requirements, but not completely. The basic function model can be further divided into more specific functional models through inheritance. Specific capacity requirements are generally given by the users according to the actual needs, and described in the military conceptual model. The function model layer of warfare model system is composed of the function model which is extracted from the operational capability requirements. The second layer in Figure 2 shows the function model layer of the weaponry model architecture. The equipment model of the entity model layer can be assembled by the functional model in function model layer. 


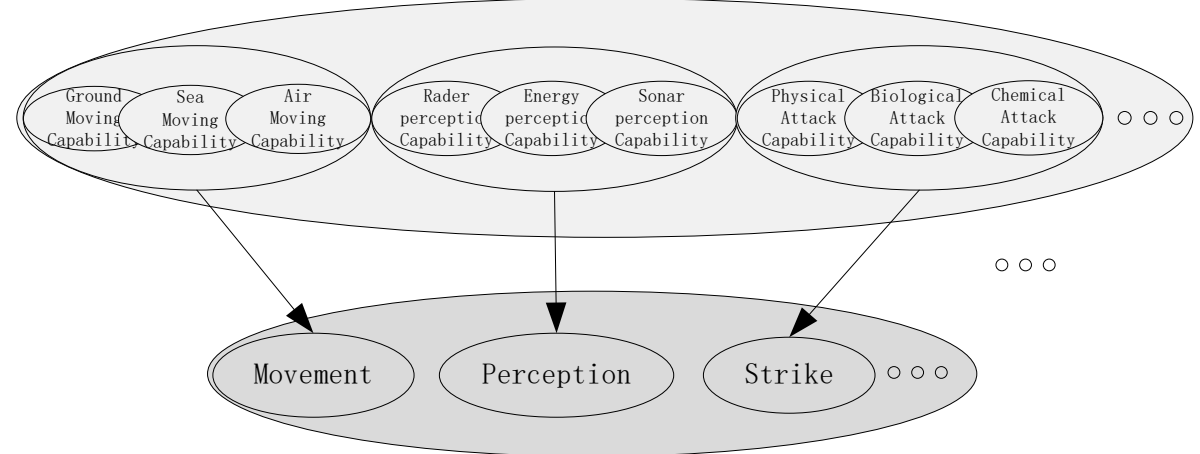

Fig. 3. The extraction of the basic function modules

The third layer is called abstract entity model layer. Because there is a case that an equipment mode assembled by another equipment model, such as early warning aircraft model can be combined by model aircraft and radar model, it is not enough to simply divide the equipment model into different functional modules. In order to ensure the flexibility of the model combination and the direct reuse of equipment model, we also need to further abstract the equipment model and functional model and what we abstract called abstract entity model. Abstract entity model is the parent of all functional models and equipment models, and the equipment model can be composed of abstract entity model, as shown in figure 4. In this design pattern, the equipment model can not only be combined with function models, but also can be combined with other equipment models. The third layer in Figure 2 shows the abstract entity model layer of the weaponry model architecture.

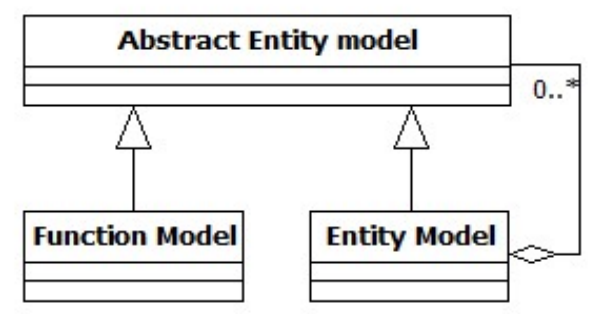

Fig. 4. The relationship about entity model, function model and abstract entity model

Based on the method above, this paper establishes a weaponry model architecture, which has three model layers: entity model layer, function model layer and abstract entity model layer. With this weaponry model architecture, we can establish weapon model by inheritance, combination and polymorphic, which can improve the efficiency of modeling, and the reusability of weapon model. It should be emphasized that the interaction between the weapon models can only be carried out in the entity model layer, and the weapon model needs to encapsulate the interface of the functional model to ensure the interaction between the weapon models is consistent with the real situation.

\section{Example}

In order to verify the flexibility of weapon modeling with weaponry model architecture and show the reusability of the model, this paper will take fighter as an example Show the modeling process based on weaponry model architecture.

Generally, the fighter is composed of the fire control radar, radar warning, navigation systems, electronic warfare systems, defense systems, communication systems, weapon systems, motion system and command and control system. According to the weaponry model architecture we can know that the fighter model class is a subclass of space-based platform model class, and space-based platform is a subclass of the abstract entity model class. At first we need to design abstract entity model, then generate space-based platform model class by inheriting the abstract entity model class, finally extend space-based platform model class to generate fighter model class. In this process, we can find that once the abstract entity model class and space-based platform model class have been designed, they can be reused by inheritance. Radar function model and 
navigation function model are subclasses of perception function model, and fire control radar model and early warning radar model can inherit from radar function model. Similarly, the radar function model and navigation function model can be reused by assembly and inheritance. The other function of models can also expand from the corresponding basic functions model. Figure 5 shows a fighter model assembled of function model. Through the process of fighter modeling, we can find that modeling of equipment model based on weaponry model architecture, can effectively realize the model assembly and model reuse.

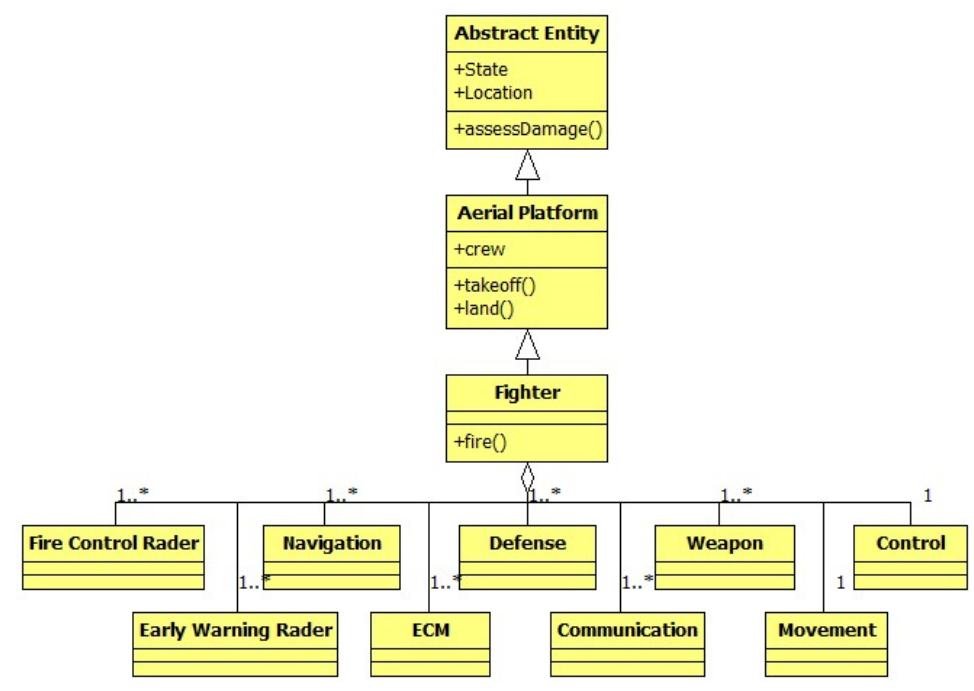

Fig. 5. Function composition of aircraft model

\section{Conclusions and Future work}

It needs formal methods for weaponry model architecture to develop composable and reusable models. However there is not mature method that can satisfy demands of model composition and reuse in the warfare simulation system. In this paper, we propose a three-layer weaponry model architecture design method, which contains equipment model layer, function model layer and abstract entity model layer. At the end of this paper, an example is given to prove that the reusability of the models and the flexibility of model developing based on the weaponry model architecture.

The equipment model is the foundation of combat simulation system. We could build military equipment model by inheritance, parametric assembly, even directly reuse, if we could construct a general weaponry model library based on this model architecture. Overall, the weaponry model architecture can greatly improve the efficiency of warfare simulation system development and reusability of models.

\section{Acknowledgement}

We appreciate the support from State Key Laboratory of High Performance Computing, National University of Defense Technology (No.201303-05) and Research Found for the Doctoral Program of Higher Education of China (No.20124307110017).

\section{References}

[1] Kedi Huang, Baohong Liu, Jian Huang, Xingping Cao, Quanjun Yin, Gang Guo, Qi Zhang, Chuanfu Zhang, Yunsheng Liu. A Survey of Military Simulation Technologies [J]. Journal of System Simulation, 2004, 16(9): 1887-1895.

[2] Lianxing Jia, Weifeng Shan, Yunjun Lu, Chenggong Zhai, Hua Liu. Research on Devel-opment of Operational Simulation [J]. Journal of System Simulation, 2007, 19(14): 3148-3157. 
[3] Xiaoyu Kang. Research on Key Technologies of Simulation Model Reusability and Composability [D]. Dalian University of Technology, 2012.

[4] Yong Peng. Research on Key Technologies for Analysis of Model's Family, Design and Implement of Its Model for Warfare Simulation [D]. National University of Defense Technology, 2011.

[5] Conwell. Joint Warfare Simulation Object Library: Joint Warfare Taxonomy [R]. Naval Command Control and Ocean Surveillance Center, San Diego, California 92153-5001.

[6] Don Bates. Joint Warfare System (JWARS) Update to the Executive Forum on Modeling and Simulation [R]. https://www.jointmodels.mil/jwars, 2001.

[7] Dudgeon Douglas E. Developing a Standard Platform Level Army Object Model [D]. Monterey, CA, USA: America Naval Postgraduate School Master Thesis, 1998.

[8] Rumbaugh, J., Blaha, M., Premerlani, W., Eddy, F., Lorenson, W. Object-oriented Modeling and Design, Prentice Hall, 1991.

[9] Andy Ceranowicz. ModSAF (Modular Semi-Automated Forces) [C] //In Proceeding of 2006 Winter Simulation Conference, San Diego, CA, 2006, 755-761.

[10] National Simulation Center. Operational Requirement Document (ORD) for War-fighters' Simulation (WARSIM) 2000 [R]. Fort Leavenworth, 1998. 